\title{
Evaluation and management of lymphadenopathy in child: When should a malignity be suspected?
}

\author{
Monica Dragomir \\ "Carol Davila" University of Medicine and Pharmacy, Bucharest, Romania \\ "Prof. Dr. Al. Trestioreanu" Oncological Institute, Bucharest, Romania
}

\begin{abstract}
The lymph nodes are normal structures and some lymph nodes can be palpable in a healthy patient. The presence of abnormal lymph nodes (lymphadenopathy) can be an indication of a serious systemic disease, and the differential diagnosis can be an ample one. The challenge for the family doctor and pediatrician is to distinguish pathological lymphadenopathies from non-pathological ones and to develop a rational approach to assessing lymph node pathology. Due to association with oncological diseases, lymphadenopathy can be a major source of anxiety for parents. It is essential to recognize the moment when the reference to the pediatric oncologist is justified. Although the discovery of lymphadenopathy sometimes raises concerns about a serious illness, it is often the result of benign infectious causes. Most patients can be diagnosed on the basis of careful history and physical examination. Localized adenopathy requires the search for a causal lesion of proximity and the evaluation of other lymph nodes to rule out generalized lymphadenopathy. Generally, lymph nodes with a diameter of more than $1 \mathrm{~cm}$ are considered abnormal. Supraclavicular adenopathies are most suggestive of malignancy. An observation period of three to four weeks is prudent in patients with limfadenopathies localized with characteristics of benignity. Generalized adenopathy always involves additional clinical investigations. In cases where lymph node biopsy is indicated, the excisional biopsy of the most affected lymph node is recommended, which will allow the pathologist to establish the diagnosis of certainty.
\end{abstract}

Keywords: lymphadenopathy, cancer, child

The normal development of lymphoid tissue is growth until puberty and then progressive atrophy in the rest of life. Normal ganglia are palpable especially in children aged 4 to 8 years. Only submandibular, axillary and inguinal nodes can be palpated in healthy people. Lymphadenopathy is more common in young children whose naïve system frequently responds to repeated infections

\section{DEFINITION}

Lymphadenopathy is the increase in lymph node size. A limph node is considered pathological at dimensions exceeding $10 \mathrm{~mm}$ in diameter. Exceptions are epitrohlear ganglia (pathologically over 5 $\mathrm{mm}$ diameter) and inguinal ganglia (pathologically over $15 \mathrm{~mm}$ diameter). The most common lymphadenopathies are secondary to infections, they are self-limiting and do not require treatment.

\section{LYMPH NODES ROLE IN IMMUNE RESPONSE}

There are approximately 600 ganglia in the human body. The lymph passes through at least one node before returning to the circulatory torrent. Related ganglia contain antigens, including partially destroyed microorganisms. Lymph contains microorganisms and cytokines from inflammatory and infected areas. Microorganisms can be processed and presented as antigens in the lymph node. Lymphocytes proliferate in the limph node. B cells mature in plasma and secrete antibodies. Infections and antigenic stimulation cause lymphadenopathy. Causes of lymph node enlargement in size are: hyperplasia, lymphocytic infiltration and tissue edema. Ganglia have numerous germinal centers with active cell proliferation. Symptoms of acute lymphadenitis reflects the response to the infection. Malignant cells can reach the lymph node and then 
other parts of the body. Anatomy of lymph drainage helps to identify causal pathological lesions.

\section{PATHOGENIC CLASSIFICATION}

Lymphadenopathy may be caused by proliferation of intrinsic lymph node cells (lymphocytes, plasmocytes, monocytes, histiocytes) or infiltration with extrinsic cells: neutrophils or malignant cells.

- The inflammatory adenopathy resulting from loco-regional inflammation is a localized response of lymphocytes and macrophages secondary to viral or bacterial infection.

- Lymphadenitis is an infection of a lymph nodes.

- Malignant adenopathy is caused by the malignant neoplastic proliferation of lymphocytes and macrophages (in leukemias and lymphomas) or infiltration of the lymph node with malignant cells from a primary tumor (lymph node metastasis).

Anamnesis and careful clinical examination are extremely important in the diagnosis of lymphadenopathy.

\section{MEDICAL HISTORY}

Questions that "narrow" the spectrum of differential diagnosis.

\section{Characteristics of lymphadenopathy}

1.1. Onset. How long has adenopathy been observed?

- Acute lymphadenopathy is most likely caused by an infectious process.

- Chronic lymphadenopathy (lasting over 4 weeks) is caused by a chronic infection or malignancy.

- Unilateral or bilateral acute lymphadenopathy is caused by streptococcal or staphylococcal infections in $40-80 \%$ of cases

- Subacute/chronic lymphadenopathies are most commonly caused by cat's claw disease, mycobacterial infection, toxoplasmosis, EBV, CMV, collagenosis, and so on.

- Associated symptomatology may suggest the etiology of lymphadenopathy

- Fever, sore throat, coughing suggests an acute upper respiratory infection

- Fever, night sweats, weight loss suggests a TB infection or lymphoma

- Unexplained fever, asthenia, arthralgia are suggestive of collagenoses

\section{Recent infections. Is there a recent infec- tion that explains lymphadenopathy?}

Suggestive for a recent infection are: respiratory symptoms, rashes, intestinal transit disorders, bone or joint pain, visual disturbances, headache.

3. Skin injuries or trauma? Cat scratches, bruises or bites of animals, open wounds, dental abscesses are signs that can help determine the cause of lymphadenopathy.

4. Infectious contact or travel. Was it stung by insects or had any infection on a trip? (Tick - Lyme disease, tularemia). Was he in contact with sick people? Was in contact or had EBV/CMV infection? Or TB?

5. Immunization status: Immunization of MMR (measles-rubella-mumps) can cause inflammatory adenopathy

6. Medication. Medicines that can cause adenopathies: carbamazepine, phenytoin, cephalosporins, penicillin, pyrimethamine

7. Allergies can cause local-regional lymphadenopathy.

8. Adolescent. Questions will be asked about drug use and sexual contacts.

9. Cats. The presence of cats in the child's entourage suggests toxoplasmosis or bartonellosis

10. Food. Ingestion of unpasteurized milk may be related to brucellosis; ingestion of meat insufficiently prepared may cause toxoplasmosis or tularemia

\section{CLINICAL EXAMINATION}

Children often have palpable lymphadenopathies in response to infections. Adenopathies that are frequently palpated up to 12 years of age are anterior cervical, inguinal, axillary cervix. The palpable nodules over $1 \mathrm{~cm}$ in diameter in the cervical region are frequently encountered in children 38$45 \%$. In most cases, it is a transient response to localized or generalized infections. Palpable lymphadenopathies in the supraclavicular region are frequently associated with malignancies (thoracic or abdominal tumors) and require immediate referral to an oncology center.

Physical examination is important in assessing fever, weight status (has lost weight recently?) and general condition. Particular attention should be paid to the region of the head and neck, abdomen and tegument.

Clinical examination of the head and neck region: we will look for scalp infections (seborrheic dermatitis, tinea capitus), conjunctival congestion, 
oropharynx for pharyngitis, dental problems, herpetic gingivostomatitis, ears for medium otitis.

Abdominal examination: look for hepato-splenomegaly and abdominal tumors (eg neuroblastoma).

Skin examination will look for any kind of eruptions, petechiae, purpura, ecchymosis (trobocytopenia!)

\section{CLINICAL EXAMINATION OF LYMPHADENOPATHY}

Palpation of lymphadenopathy should evaluate:

1. The size of lymphadenopathy. It is not decisive for the diagnosis of malignancy.

2. Location. Cervical and axillary adenopathy suggests cat's claw disease, cervical adenopathy suggests mononucleosis, inguinal adenopathy may appear related to sexually transmitted diseases. Generalized adenopathies occur in systemic diseases

3. Lymphadenopathy blocks are a group of nodes that seem connected and mobilized together. They are characteristic of sarcoidosis, $\mathrm{TB}$, lymphatic metastases in a solid tumor, malignant lymphoma.

4. Consistency. Firm, tough consistency is suggestive of carcinomatous metastasis, fermelastic consistency is characteristic of malignant lymphoma, low consistency characterizes infectious and inflammatory lymphadenopathies. Fluctuating consistency suggests suppurative adenitis. Adenopathies with "small shot" consistency under the skin characterize viral infections.

5. Sensitivity. Does not always make the difference between benign/malignant. Pain may be the consequence of rapid ganglion enlargement and capsule distention, inflammation or suppuration, as well as bleeding in the necrotic center of a malignant lymphnode

\section{Risk of malignancy of a lymphadenopathy}

- Low malignancy risk: less than 1-2 cm in diameter, cervical, inguinal or axillary location, associated erythema, pain sensitivity, local heat, fluctuation.

- Increased risk of malignancy: occipital, auricular, supraclavicular, mediastinal, epitrochlear, posterior cervical localization, firm consistency, lack of pain, diameter greater than $2 \mathrm{~cm}$, association with systemic symptomatology.

\section{DIFFERENTIAL DIAGNOSIS}

1. GENERALISED LYMPHADENOPATHY: more than 2 non-contiguous lymph nodes

Infectious causes:

- Viral infections: respiratory, varicella, measles, rubella, hepatitis, HIV, EBV, CMV, adeno

- Bacterial infections: syphilis, brucellosis, TB, sepsis

- Mycosis: histoplasmosis, coccidiosis

- Toxoplasmosis

Non-infectious inflammatory diseases: sarcoidosis, rheumatoid arthritis, lupus, storage diseases (Niemann Pick, Gaucher)

Malignancies: leukemias, lymphomas, neuroblastoma

Medicinal reactions: phenytoin, allopurinol

Hyperthyroidism

2. LOCALIZED LYMPHADENOPATHY: a lymph node or contiguous lymphadenopathy

Head and neck region:

Infectious causes: Viral respiratory infections, mononucleosis, group A streptococcal pharyngitis, acute bacterial lymphadenitis, Kawasaki disease, rubella, cat's claw disease, toxoplasmosis, TB, and atypical mycobacterial infections

Malignant causes: $1 / 4$ of child malignancy occurs in the head and neck region

- Children under 6 years old: leukemias, lymphomas, neuroblastoma, rhabdomyosarcoma

- Children over 6 years old: Hodgkin's lymphoma and non-Hodgkin's lymphoma

Mediastinal region: lymphadenopathy can not be palpated directly. The evaluation is made indirectly by the presence of the supraclavicular lymph nodes. Mediastinal adenopathies may be accompanied by coughing, dysphagia, haemoptysis within a superior vena cava syndrome (medical emergencies!)

Etiology: acute leukemia, malignant lymphoma, sarcoidosis, cystic fibrosis, TB, histoplasmosis.

The supraclavicular region (associated with serious diseases): lymphoma, TB, histoplasmosis

Axillary region: Local infections, cat's claws, ARJ, suppurative hydrosadenitis, non-Hodgkin's lymphomas.

Abdomen: Lymphdenopathies can manifest as abdominal pain, lumbar pain, frequency micturition, constipation, intestinal obstruction by intrusion. It may occur in mesenteric lymphadenitis or malignant lymphoma.

Inguinal: local infections, diaper dermatitis. 
It is very important to exclude malignancy. In the absence of any signs and/or symptoms suggestive of a malignant process, adenopathy should be carefully monitored until disappearance. If adenopathy does not resolve in 4 weeks, it is considered chronic and only lymph node biopsy can rule out malignancy.

\section{LYMPH NODE BIOPSY INDICATIONS}

- Increase in size of adenopathy after 2-3 weeks of antibiotic treatment

- Lymph Nodes that did not grow in size but did not decrease in size in 6-8 weeks

- Lymphadenopathies associated with any mediastinal abnormality in chest radiography

- Lymphadenopathy associated with weight loss, hepato- splenomegaly, fever without obvious cause and/or nocturnal sweating

- Lymphadenopathies localized, epitrochlear, supraclavicular or auricular posterior

\section{LYMPHADENOPATHY IN CHILDHOOD MALIGNANCIES}

Acute leukemias: Lymphadenopathies are present at onset in $50 \%$ of cases; are usually large and grow rapidly in size. Other associated clinical manifestations: palpitations, fever, patches, generalized pain, bruising, hepatosplenomegaly. Modified blood count: pancytopenia, the number of leukocytes may be normal or increased. Onset with acute infection that does not progress favorably to antibiotic treatment.

Hodgkin's lymphoma. Rarely in children under 4 years of age. Clinical appearance of adenopathy: "like walnuts in the bag". Painless, strong, mobile lymph node, sometimes with inflammatory phenomena. Systemic symptomatology associated in $30 \%$ of cases: fever, anorexia, weight loss, pruritus.

Non-Hodgkin's lymphoma. Onset more suddenly, apparently in full health. It may occur in

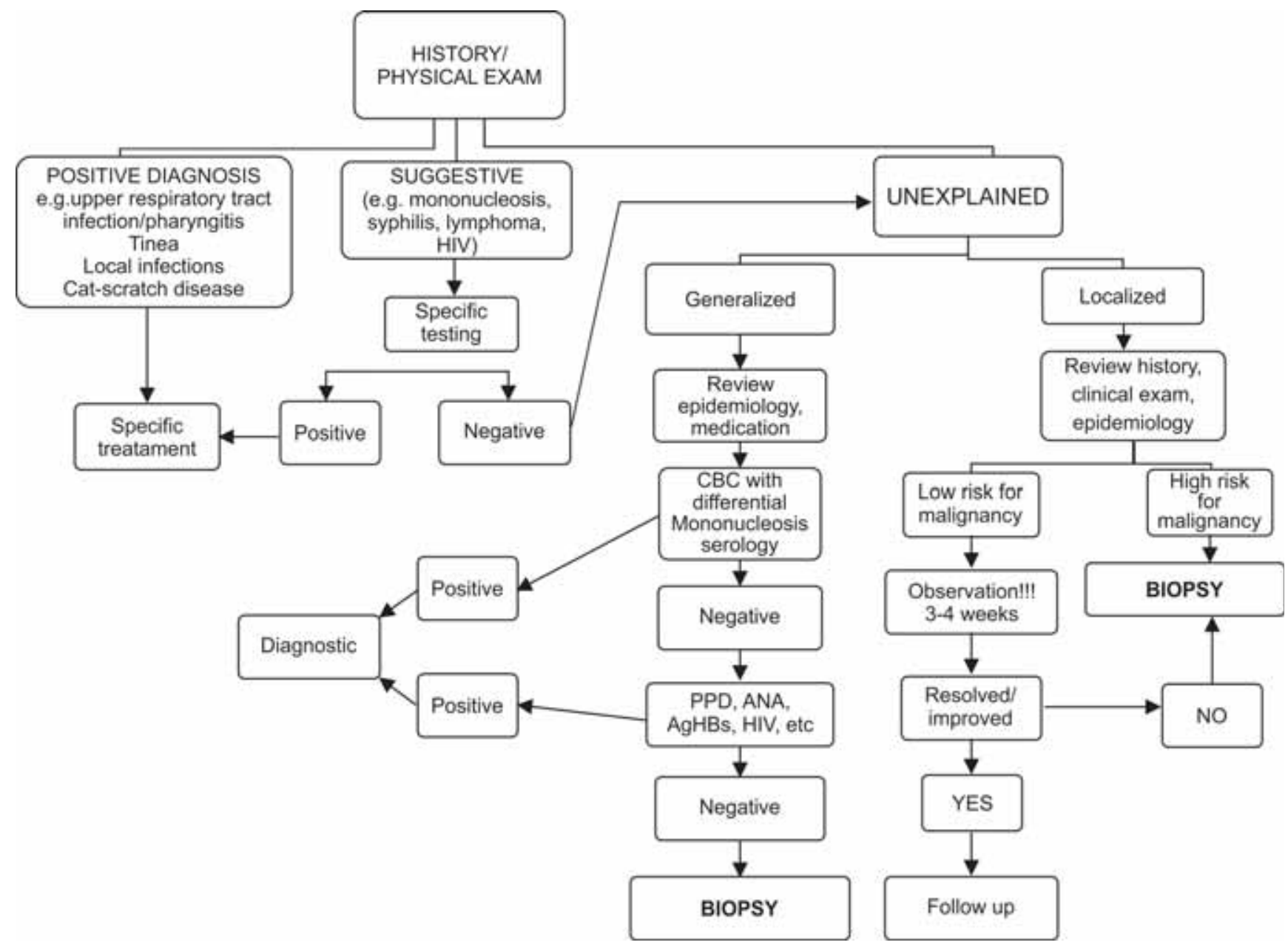

FIGURE 1. Diagnostic approach to lymphadenopathy 
young infants (abdominal lymphoma). More commonly in children $<10$ years of age. Often the stage of diagnosis is advanced. Localizations at onset: abdomen $45 \%$; mediastinum $25 \%$; head and neck 10-20\%; superficial ganglia 5-10\%; other locations $5-10 \%$.

Neuroblastoma with onset in the lymph nodes of the sympathetic nervous system. In the cervical region it causes Horner syndrome (miosis, palpebral ptosis, enophtalmos).

Other malignancies: lymph node metastases from rhabdomyosarcoma, rhinopharinx carcinoma, thyroid carcinoma.

\section{ALGORITHM TO ASSESS A PATIENT WITH LYMPHADENOPATHY}

What is the right attitude to an lymphadenopathy discovered in a child during the clinical examination, regardless of the cause for which the family requested the medical consultation?

Following careful history and clinical examination, we are faced with three situations (Fig. 1):

1. Diagnosis of the condition causing adenopathy with benignity characteristics is established: acute respiratory infection, pediculosis, local infection, cat's claw disease, etc. Proper treatment is initiated. The patient should be monitored until healing the disease and the remission of adenopathy.

2 . The diagnosis suggested by clinical examination and anamnesis is specific infection: mononucleosis, HIV, syphilis. Serological tests are performed and, if the diagnosis is confirmed, etiological treatment is instituted. The patient is monitored until healing and the remission of adenopathy. In cases where the specific tests are negative, we are facing a "adenopathy without known cause"

3. Lymphadenopathies without known cause by first-degree investigations. In this situation, the patient's medical history, the medication received and the clinical examination are re-evaluated, the epidemiological context is re-evaluated.

There are two possible situations:

3.1. Generalized adenopathy. Complete blood count with smear and mononucleosis test will be performed. The positive test will confirm the diagnosis and antibiotic treatment will be established. If the test is negative, other serological tests may be performed that may indicate the pathological context in which lymphadenopathies occurred: antinuclear antibodies, PPD, HIV test, Ag.HBS. If positive tests establish the diagnosis, appropriate treatment is initiated. If the performed tests are negative, ganglion biopsy is recommended.

\subsection{Localized adenopathy}

If adenopathy is part of the "low malignancy risk" category (based on the above mentioned criteria), the patient remains in observation for 3-4 weeks. If, during this time, partial remission or complete remission of adenopathy is observed, we keep the child in observation without taking any other therapeutic attitude or affecting other investigations. If adenopathy is maintained or increased within 3-4 weeks, lymph node biopsy is recommended.

If adenopathy is part of the "increased risk of malignancy", lymph node biopsy is recommended without further investigation that could delay the diagnosis.

\section{REFERENCES}

1. Brigger M.T., Cunningham M.J. Malignant cervical masses in children. Otolaryngol Clin North Am. 2015 Feb; 48(1):59-77.

2. Chesney P.J. Cervical adenopathy. Pediatr Rev. 1994 Jul; 15(7):276-84

3. Citak E.C., Koku N., Demirci M., Tanyeri B., Deniz H. A retrospective chart review of evaluation of the cervical lymphadenopathies in children. Auris Nasus Larynx. 2011 Oct; 38(5):618-21

4. Ferrer R. Lymphadenopathy: Differential Diagnosis and Evaluation. Am Fam Physician. 1998 Oct 15; 58(6):1313-1320.

5. Friedmann A.M. Evaluation and management of lymphadenopathy in children. Pediatr Rev. 2008 Feb; 29(2):53-60

6. Jackson M.A., Chesney P.J. Lymphatic system and generalized lymphadenopathy. In: Principles and Practice of Pediatric Infectious Diseases, 4th ed, Long SS, Pickering LK, Prober CG (Eds), Elsevier Saunders, Edinburgh 2012. p.127.

7. Knight P.J., Mulne A.F., Vassy L.E. When is lymph node biopsy indicated in children with enlarged peripheral nodes? Pediatrics 1982; 69:391.

8. Leung A.K., Robson W.L. Childhood cervical lymphadenopathy. J Pediatr Health Care. 2004 Jan-Feb; 18(1):3-7.

9. O'Malley D.P., Grimm K.E. Reactive lymphadenopathies that mimic lymphoma: entities of unknown etiology. Semin Diagn Pathol. 2013 May; 30(2):137-45

10. Penn E.B. Jr., Goudy S.L. Pediatric inflammatory adenopathy. Otolaryngol Clin North Am. 2015; 48:137-151.

11. Rajasekaran K., Krakovitz P. Enlarged neck lymph nodes in children. Pediatr Clin North Am. 2013 Aug; 60(4):923-36.

12. Rosenberg T.L., Nolder A.R. Pediatric cervical lymphadenopathy. Otolaryngol Clin North Am. 2014; 47:721-731.

13. Shashi S. Lymphadenopathy. Pediatr Rev., 2008, May, 34(5) 